REVISTA DE ESTUDIOS E INVESTIGACIÓN EN PSICOLOGÍA Y EDUCACIÓN

\title{
Fatores associados ao rendimento acadêmico na formação inicial de professores
}

\section{Factors associated with academic performance in the initial teacher training}

\author{
Grasiany Sousa de Almeida (D) http://orcid.org/0000-0003-2725-5161 \\ Universidade Estadual do Ceará (UECE): http://www.uece.br/ \\ Leandro Araujo de Sousa (D) http://orcid.org/0000-0002-0482-2699 \\ Instituto Federal de Educação, Ciência e Tecnologia do Ceará (IFCE): https://ifce.edu.br/ \\ Adriana Eufrásio Braga (iD) http://orcid.org/0000-0001-5163-209X \\ Universidade Federal do Ceará (UFC): http://www.ufc.br/ \\ José Airton de Freitas Pontes Junior (iD http://orcid.org/0000-0003-2045-2461 \\ Universidade Estadual do Ceará (UECE): http://www.uece.br/ \\ Fortaleza, Ceará - Brasil
}

\footnotetext{
Pesquisa financiada com bolsa de pesquisa da Coordenação de Aperfeiçoamento de Pessoal de Nível Superior. Bolsista: Grasiany Sousa de Almeida.

Correspondência relativa a este artigo: Leandro Araujo de Sousa - leandro.sousa@ifce.edu.br
} 


\title{
Resumo
}

O contexto socioeconômico e as características da Instituição de Ensino Superior podem desempenhar um papel importante no desempenho académico dos estudantes. Uma melhor compreensão destes fatores pode ajudar a focalizar e orientar as políticas públicas e a atribuição de recursos. O objetivo deste estudo era avaliar se o desempenho acadêmico de estudantes dos cursos de licenciatura em matemática está associado com o contexto socioeconômico e características da Instituição de Ensino Superior. O estudo incluiu uma amostra de 10669 estudantes concluintes dos cursos de Licenciatura em Matemática. Os dados foram a nota na prova e as variáveis do "Questionário do Estudante" do Exame Nacional de Desempenho dos Estudantes do Brasil. Foi utilizada a regressão multinível para análise dos dados. O modelo ajustado obtido da análise mostra que as variáveis renda familiar e bolsa de estudo estão fortemente associadas ao desempenho académico dos estudantes na formação inicial de professores em Matemática. Verificou-se também que outras variáveis tiveram um efeito significativo no modelo.

Palabras clave: educação superior; formação de professores; avaliação educacional; desempenho acadêmico

\begin{abstract}
Socioeconomic background and the characteristics of the Higher Education Institution characteristics can play an important role in students' academic performance. A better understanding of these factors can help to focus and orient public policies and the allocation of resources. The aim of this study was to assess the influence of socioeconomic background and Higher Education Institution characteristics on the students' academic performance. The study comprised a sample of 10,669 students from the last year of the Mathematics Degree. Data were the test scores and variables from the "Student Questionnaire" of the Brazilian National Student Performance Exam. The data were analyzed using multilevel regression. The fitted model obtained from the analysis shows that the variables white skin color, family income and scholarship are closely associated with the academic performance of Mathematics undergraduates. Other variables were also found to have a significant effect on the model.
\end{abstract}

Keywords: higher education; teacher training; educational assessment; academic performance 
Nos últimos anos cresceu a oferta de cursos e matrículas para os cursos de formação de professores no Brasil (Camargo e Medeiros, 2018; Maués e Camargo, 2014; Maués et al., 2015; Maués e Souza, 2013). Dessa forma, mecanismos de acompanhamento como o Sistema Nacional de Avaliação do Ensino Superior (Sinaes) são importantes para tomadas de decisão pelos gestores públicos.

Como parte desse sistema, o Exame Nacional de Desempenho dos Estudantes ( ENADE) é uma prova aplicada aos estudantes dos cursos de graduação com o objetivo de mensurar os conhecimentos gerais e conhecimentos específicos referente ao curso. Entretanto, o desempenho dos estudantes avaliados em um exame pode ser afetado por vários fatores socioeconômicos, psicológicos e institucionais (Álvaro Page et al., 1990).

Apesar disso, poucos estudos investigam a influência desses contextos no desempenho acadêmico durante o processo de formação inicial de professores. No Brasil, há alguns estudos realizados na área de ciências contábeis, administração e pedagogia (Medeiros Filho et al., 2019). Entre os estudos, analisa-se apenas condições isoladas, como as características institucionais, infraestrutura, adequação do espaço pedagógico, número de alunos, qualidade, adequação aos equipamentos de laboratórios, entre outros (Andriola, 2009) e diferenças entre sexo (Silva et al., 2010). Estudantes bolsistas também apresentam melhor rendimento acadêmico (Araujo et al., 2018).

Da mesma forma, em estudo de revisão de literatura são encontradas poucas teses e dissertações que tratem de analisar o desempenho dos estudantes no ENADE entre 2004 e 2010 (Molck e Calderón, 2014). Após esse período, algumas pesquisas de teses e dissertações investigam essas variáveis. $O$ desempenho de estudantes de Licenciatura em Educação Física no ENADE 2017 foi melhor entre os de cor branca ou amarela, que cursaram o ensino médio profissionalizante, que os pais possuíam escolaridade com nível de pós-graduação e que não possuíam vínculo empregatício (Medeiros Filho, 2019). Na análise de estudantes de pedagogia no exame do mesmo ano, características como maior escolaridade dos pais, recebimento de bolsas, que estudavam em instituições públicas e em cursos presenciais estiveram positivamente relacionadas ao desempenho (Costa, 2019). Ao investigar o desempenho no ENADE 2013 de 12 cursos de graduação, nenhum desses de licenciatura, foi identificado relação positiva com a renda familiar dos pais, nível de dedicação aos estudos, recebimento de bolsas, se a instituição é pública, entre outras (Araújo, 2017). No curso de Administração o desempenho dos estudantes no exame do ano de 2012 esteve positivamente associado com a escolaridade dos professores, quando o docente tem regime de dedicação integral a instituição, a quantidade 
de docentes por curso e se a instituição é pública (Brito, 2015). Desses resultados, é possível verificar que investigações sobre quais variáveis estão associadas ou conseguem explicar o desempenho de estudantes de cursos de formação de professores são escassos.

Diante disso, a compreensão de variáveis que podem estar a associadas ao desempenho de estudantes de licenciatura pode auxiliar nas tomadas de decisão para melhorar a formação dos professores, que tem sido apontada como um dos importantes fatores para a melhoria da qualidade da educação (Bauer et al., 2017).

Dessa forma, esta pesquisa foi orientada pelo seguinte problema: a aprendizagem dos estudantes da formação inicial de professores de matemática pode ser explicada por suas características socioeconômicas e pelos fatores institucionais? Diante disso, a pesquisa teve o objetivo de analisar se o desempenho acadêmico de estudantes dos cursos de licenciatura em matemática está associado com o contexto socioeconômico e características da instituição de Ensino Superior.

\section{O que pode explicar o desempenho acadêmico no ensino superior?}

Quando nos referimos a fatores de contexto como condicionantes do rendimento acadêmico é possível elencar alguns estudos empíricos realizados no âmbito do ensino superior. Nesses estudos, fatores como status socioeconômico, etnia/cor, sexo, nível econômico, escolaridades dos pais, infraestrutura e as variáveis relacionadas a instituição podem ser condicionantes no desempenho escolar (Garbanzo Vargas, 2007; Andriola, 2009; Barbosa et al., 2011; Thiele et al., 2016; Barrios Gaxiola e Frías Armenta, 2016; Rocha et al., 2018; Oliveira e Silva, 2018; Araujo et al., 2018).

A influência da variável sexo no desempenho em matemática é bastante controverso e passível de discussão, porém estudos revelam que os alunos do sexo masculino tendem a ter melhor predisposição a essa disciplina do que os do sexo feminino (Garbanzo Vargas, 2007; Barahona U, 2014). No entanto, é necessário compreender os contextos de aprendizagem para melhor explicação dessas diferenças. Por exemplo, as expectativas em relação a conclusão do curso, assim como as expectativas relacionadas a ascensão social é maior nos familiares de estudantes do sexo masculino (Mascarenhas e Roazzi, 2015), o que pode explicar esse contexto mais favorável a aprendizagem desse grupo.

No que se refere as características de cor ou raça, alguns estudos indicam que alunos brancos têm melhores rendimentos do que alunos declarantes pretos, pardos e indígenas (Rodrigues et al., 2015; Rocha et al., 2018; Silva et al., 2020). Essas diferenças podem estar 
associadas ao desenvolvimento histórico e cultural desfavorável a imersão da população de cor ou raça preta e pardas nos ambientes educacionais, principalmente no Ensino Superior. Isso também está associado as condições socioeconômicas desfavoráveis desse grupo.

Quando bem estruturado economicamente, os alunos tendem a ter acesso a bens e serviços, tais como frequentar boas instituições, possuir melhor infraestrutura em casa, realizar viagens de estudo (Oliveira e Silva, 2018). Partindo dessa discussão, entende-se que alunos oriundos de famílias economicamente estruturadas têm rendimentos superiores daqueles que não dispõem de melhores condições (Knop e Collares, 2019; García-González e Skrita, 2019; Rodrigues et al., 2017; Thiele et al., 2016; Crepalde e Silveira, 2016; Oliveira e Melo-Silva, 2010).

Além das condições socioeconômicas adequadas serem favoráveis ao aprendizado, a escolaridade dos pais se associa positivamente ao bom desempenho. Estudos apresentam evidências de que essa variável de fato influencia no desempenho dos alunos que tende a ser melhor quando os pais possuem maior escolaridade (Costa, 2019; Brito, 2015; Reyes Carreto et al., 2014; Rocha et al., 2018; Knop e Collares, 2019; García-González e Skrita, 2019). É importante observar que essas duas variáveis, condições socioeconômicas e escolaridade dos pais podem estar relacionadas, uma vez que escolaridade maior está associada a maior renda.

Embora os insumos familiares exerçam forte influência no aprendizado dos estudantes, essas variáveis são pouco flexíveis a alterações mediante políticas públicas. Nesse sentido, a instituição de ensino também tem papel importante no aprendizado dos estudantes. Assim, alguns estudos apresentam evidências de que as instalações físicas, a participação dos discentes em projetos de pesquisa e extensão, assim como a participação estudantes de licenciatura em programas de bolsa como o Programa Institucional de Bolsas de Iniciação à Docência (Pibid) e o Programa Residência Pedagógica (PRP), em que recebem uma quantia em dinheiro e atuam como docentes nas escolas enquanto estão estudando com a supervisão de professores experientes, estão positivamente associados ao maior rendimento acadêmico e ao desempenho nas atividades docentes (Andriola, 2009; Araujo et al., 2018; Souza et al., 2020). Em estudos foi observado forte associação entre os indicadores de gestão, como por exemplo, o custo por aluno, e o desempenho dos estudantes (Barbosa et al., 2011; Lavor et al., 2015).

Diante disso, os estudos indicam que variáveis relacionadas tanto ao contexto socioeconômico dos estudantes, como cor ou raça, renda e escolaridade dos pais, como as variáveis relacionadas a instituição de ensino, como programas e projetos institucionais, podem ser explicativas do rendimento acadêmico. Por tanto, este estudo buscou analisar se há associações entre essas variáveis em estudantes de licenciatura em matemática. 


\section{Método}

\section{Participantes}

Foram utilizados os microdados disponíveis de livre acesso no site do Instituto Nacional de Estudos e Pesquisa Anísio Teixeira (Inep). Neste estudo foram utilizados os dados de 10669 estudantes de licenciatura em matemática de 426 cursos que participaram do ENADE aplicado no ano de 2017 e que estivem presentes com situação regular na prova. A média de idade dos estudantes é de 29.7 ( \pm 8.65) com idade mínima de 16 e máxima de 72 anos. Outras características dos participantes estão na Tabela 1.

\section{Tabela 1}

Características dos estudantes de Licenciatura em Matemática ( ENADE 2017)

\begin{tabular}{|c|c|c|c|}
\hline Variáveis & Níveis & $\mathrm{n}$ & $\%$ \\
\hline \multirow[t]{2}{*}{ Sexo } & Masculino & 5630 & 47.2 \\
\hline & Feminino & 5039 & 52.8 \\
\hline \multirow[t]{6}{*}{ Cor-Raça } & Branca & 4461 & 41.9 \\
\hline & Preta & 1121 & 10.5 \\
\hline & Amarela & 261 & 2.4 \\
\hline & Parda & 4528 & 42.4 \\
\hline & Indígena & 46 & 0.4 \\
\hline & Não Declarou & 252 & 2.4 \\
\hline \multirow[t]{7}{*}{ Renda familiar } & Até 1.5 salário mínimo & 3315 & 31.1 \\
\hline & De 1.5 a 3 salários mínimos & 3449 & 32.3 \\
\hline & De 3 a 4.5 salários mínimos & 2052 & 19.2 \\
\hline & De 4.5 a 6 salários mínimos & 864 & 8.1 \\
\hline & De 6 a 10 salários mínimos & 709 & 6.6 \\
\hline & De 10 a 30 salários mínimos & 267 & 2.5 \\
\hline & Acima de 30 salários mínimos & 13 & 0.1 \\
\hline \multirow[t]{6}{*}{ Escolaridade do pai } & Nenhuma & 1324 & 12.4 \\
\hline & Ensino Fundamental: 10 ao 5o ano & 4333 & 40.6 \\
\hline & Ensino Fundamental: 60 ao 9o ano & 1733 & 16.2 \\
\hline & Ensino Médio & 2280 & 21.4 \\
\hline & Ensino Superior - Graduação & 749 & 7.0 \\
\hline & Pós-graduação & 250 & 2.3 \\
\hline \multirow[t]{6}{*}{ Escolaridade da mãe } & Nenhuma & 854 & 8.0 \\
\hline & Ensino Fundamental: 10 ao 5음 ano & 3650 & 34.2 \\
\hline & Ensino Fundamental: 6ㅇ ao 9ㅇa ano & 1846 & 17.3 \\
\hline & Ensino Médio & 2853 & 26.7 \\
\hline & Ensino Superior - Graduação & 918 & 8.6 \\
\hline & Pós-graduação & 548 & 5.1 \\
\hline \multirow[t]{4}{*}{ Bolsas } & Sem bolsa & 4925 & 46.2 \\
\hline & Auxílio & 1804 & 16.9 \\
\hline & Pesquisa & 3810 & 35.7 \\
\hline & Intercâmbio & 130 & 1.2 \\
\hline \multirow[t]{2}{*}{ Instituição } & Pública & 6577 & 61.66 \\
\hline & Privada & 4092 & 38.4 \\
\hline \multirow[t]{2}{*}{ Modalidade } & Presencial & 7838 & 73.5 \\
\hline & Educação à distância & 2831 & 26.5 \\
\hline
\end{tabular}

Nota: Elaboração dos autores 


\section{Instrumentos}

A prova do ENADE é composta por um componente de formação geral, com dois itens discursivos e oito de múltipla escolha, constituindo $25 \%$ da prova; e outro componente de formação específica, com três itens discursivos e 27 itens de múltipla escolha, somando $75 \%$. 0 questionário socioeconômico é composto por 68 perguntas que visam identificar o perfil do estudante que vai realizar o exame.

\section{Análise estatística}

A análise de dados tomou como variável dependente (contínua) o escore dos estudantes no exame que varia em uma escala de 0 a 100. Estatísticas descritivas foram utilizadas para a caracterização dos estudantes. Para todas análises utilizamos o pacote Ime4 (Bates et al., 2015) no software $R$. Considerando que as variáveis possuem uma estrutura hierárquica, com variáveis ao nível do aluno e variáveis ao nível da instituição, inicialmente analisou-se a viabilidade da aplicação de um modelo de regressão multinível (Marôco, 2018).

O "modelo hierárquico" ou "modelo multinível" caracteriza-se como estrutura de modelo estatístico que melhor se ajusta a uma realidade em que as variáveis estão dispostas em níveis ou hierarquias. Esses modelos são importantes e usados em dados agrupados de maneira hierárquica (Ker, 2014; Marôco, 2018). Para a primeira etapa obtivemos a variância entre cursos e dentro dos cursos para calcular o índice de correlação intreclasse (ICC). Marôco (2018) indica que valores acima de 0,10 indicam efeito considerável dos grupos. Também utilizamos o Likelihood Ratio Tests (LRT), que quando significativo $(p \leq .05)$ é indicativo de efeito relevante dos grupos.

Para análise seguimos uma estratégia exploratória de Hox et al. (2018) que parte de um modelo mais simples e adicionando parâmetros (buttom-up), testando sua significância comparando com o modelo anterior. Esta estratégia é composta por 5 etapas: i) modelo apenas com o intercepto (intercept-only model); ii) modelo com todas as variáveis do nível mais baixo (model with all lower-level explanatory variables fixed); iii) modelo com as variáveis exploratórias do nível superior inseridas (higher-level explanatory variables); iv) modelo com variáveis do primeiro nível com efeito aleatório entre grupos (random coefficient model); v) modelo com efeitos de interação entre variáveis de níveis diferentes (cross-level interactions model).

Para cada etapa analisamos as significâncias dos parâmetros para cada variável $(p \leq .05)$ e retiramos nas análises seguintes. O LRT e o índice de Deviance foram utilizados para análise do ajuste do modelo. $O$ primeiro indica bom ajuste quando significativo $(p \leq .05)$ e o segundo 
quando apresenta valor menor em comparação ao modelo anterior. Foram consideradas como preditores relevantes as variáveis que apresentassem valores de $p \leq .05$ e coeficientes padronizados $\geq .10$ (Marôco, 2018).

\section{Resultados}

A análise inicial ocorreu com a estimação do modelo nulo, ou seja, sem nenhuma variável preditiva no modelo para verificar o quanto da variância da nota no exame é decorrida do efeito de grupo, neste caso específico, do curso. Na estimação desse modelo obtemos a variância entre grupos e a variância dentro dos grupos. O modelo nulo (M0, intercept-only model) apresenta um intercepto de 40.16, que é a grande média entre todos os participantes. A variância dentro do grupo é de 137.82 e entre grupo é de 45.95 . Considerando esses valores, o modelo indica que $25.1 \%$ da variância da nota no exame se deve as diferenças entre cursos e é significativa (Deviance $=83651, \mathrm{LRT}=1802, p \leq .05)$. Estes valores indicam a presença de efeito significativo das instituições nas diferenças das pontuações dos estudantes no ENADE, o que justifica a utilização do modelo multinível. Os valores dos parâmetros para os modelos testados estão na Tabela 2.

No modelo 1 (M1, model with all lower-level explanatory variables fixed) foram inseridas apenas variáveis do nível mais baixo (nível 1, aluno). Para este modelo, as variáveis dummy para Cor-Raça "Não Declarou" e "Amarela" não apresentam efeito significativo em relação a referência "Branca". Na etapa seguinte (M2, higher-level explanatory variable) foram inseridas as variáveis referentes ao nível 2, tipo de "Instituição", tendo a categoria "instituição pública" como referência e "Modalidade" com a categoria "educação a distância" como referência. Esta última não apresentou efeitos significativos na explicação da nota no exame. Após o ajuste desse modelo, testamos coeficientes de inclinação aleatórios (M3, random coefficient model) para as variáveis do nível 1 entre os cursos (nível 2). Para este modelo o melhor ajuste foi obtido com as variáveis "Renda familiar" e "Sexo" com inclinações aleatórias no nível 2. Na última etapa foram testados efeitos de interação entre variáveis de níveis diferentes (M4, cross-level interactions model). Apresentou efeito significativo a interação entre a dummy para a variável bolsa de "Pesquisa" (nível 1) e tipo de "Instituição" (Nível 2). Este modelo foi o que apresentou melhor ajuste em comparação aos modelos anteriores, tanto considerando o critério do LRT como pelo critério de Deviance.

É importante registrar que a variância dentro dos grupos, ou seja, a variância entre os estudantes não diminuiu consideravelmente, passando de 137.82 para 129.26 no modelo 
melhor ajustado nesse estudo. Isso pode ter ocorrido porque, apesar de todas as variáveis apresentarem efeitos significativos ( $p \leq .05)$, considerando os coeficientes padronizados, apenas as variáveis "Renda familiar" (.13) e bolsa de "Pesquisa" (.12) apresentaram coeficientes acima de .10, como recomenda Marôco (2018). As variáveis do tipo de "Instituição", dentre as demais foi a que apresentou maior coeficiente padronizado (.08).

\section{Tabela 2}

Modelos com variáveis exploratórias.

\begin{tabular}{|c|c|c|c|c|}
\hline \multirow[b]{2}{*}{ Efeitos fixos } & $M 1$ & M2 & M3 & M4 \\
\hline & Coef. (EP) & Coef. (EP) & Coef. (EP) & Coef. (EP) \\
\hline Intercepto & $34.13(.44)^{*}$ & $33.37(.93)^{*}$ & $34.70(.48)^{*}$ & $34.49(.49)^{*}$ \\
\hline Sexo (Ref: feminino) & $1.50(.23)^{*}$ & $1.50(.23)^{*}$ & $1.67(.25)^{*}$ & $1.68(.25)^{*}$ \\
\hline \multicolumn{5}{|l|}{ Cor-Raça (Ref: branca) } \\
\hline Preta & $-2.61(.41)^{*}$ & $-2.53(.40)^{*}$ & $-2.51(.40)^{*}$ & $-2.52(.40)^{*}$ \\
\hline Amarela & $-1.44(.75)$ & --- & --- & --- \\
\hline Parda & $-1.72(.27)^{*}$ & $-1.65(.26)^{*}$ & $-1.63(.26)^{*}$ & $-1.64(.26)^{*}$ \\
\hline Indígena & $-3.59(1.73)^{*}$ & $-3.52(1.73)^{*}$ & $-3.44(1.72)^{*}$ & $-3.48(1.72)^{*}$ \\
\hline Não Declarou & $-.29(.76)$ & --- & --- & --- \\
\hline Renda familiar & $1.36(.10)^{*}$ & $1.40(.10)^{*}$ & $1.33(.12)^{*}$ & $1.34(.12)^{*}$ \\
\hline Escolaridade do Pai & $.61(.11)^{*}$ & $.61(.11)^{*}$ & $.61(.11)^{*}$ & $.59(.11)^{*}$ \\
\hline Escolaridade da Mãe & $.58(.10)^{*}$ & $.57(.10)^{*}$ & $.58(.10)^{*}$ & $.58(.10)^{*}$ \\
\hline \multicolumn{5}{|l|}{ Bolsas (não) } \\
\hline Auxílio & $.91(.36)^{*}$ & $.75(.36)^{*}$ & $.81(.36)^{*}$ & $.77(.36)^{*}$ \\
\hline Pesquisa & $3.73(.30)^{*}$ & $3.55(.30)^{*}$ & $3.60(.30)^{*}$ & $3.99(.34)^{*}$ \\
\hline Intercâmbio & $5.65(1.05)^{*}$ & $5.65(1.05)^{*}$ & $5.71(1.05)^{*}$ & $5.74(1.05)^{*}$ \\
\hline Instituição (Ref: pública) & --- & $-2.23(.64)^{*}$ & $-2.02(.62)^{*}$ & $-1.55(.65)^{*}$ \\
\hline Modalidade (Ref: ead) & --- & $1.68(.90)$ & --- & --- \\
\hline Instituição*Bolsa/pesquisa & --- & --- & --- & $-1.49(.67)^{*}$ \\
\hline \multicolumn{5}{|l|}{ Efeitos aleatórios } \\
\hline Variância (nível 1) & $130.65(11.43)$ & $130.59(11.43)$ & $129.26(11.37)$ & $129.26(11.37)$ \\
\hline Variância (nível 2) & $27.16(5.21)$ & $26.42(5.14)$ & $19.18(4.38)$ & $12.18(4.38)$ \\
\hline Correlação intraclasse & .17 & .17 & .13 & .13 \\
\hline Variância (Renda Familiar) & --- & --- & $.83(.91)^{*}$ & $.83(.91)^{*}$ \\
\hline Variância (Sexo) & --- & --- & $1.62(1.27)^{*}$ & $1.62(1.27)^{*}$ \\
\hline Deviance & 82924 & 82911 & 82882 & 82878 \\
\hline LogLikel (LRT) & $-41462 *$ & $-41455^{*}$ & $-41441 *$ & $-41439 *$ \\
\hline
\end{tabular}

${ }^{*} p \leq .05$

Nota: Ref $=$ Categoria da variável de referência. Coef. $=$ Coeficiente de regressão padronizado. $E P=$ Erro padrão.

Diante disso, os resultados desse estudo indicam que o rendimento acadêmico no ensino superior, nesse caso específico, em um curso de formação de professores de matemática, pode ser influenciado por características socioeconômicas (Renda Familiar), por um lado, e por intervenções de políticas de permanência em êxito (bolsas de pesquisa), por outro. As demais variáveis como "Sexo", "Cor-Raça", "Escolaridade do pai", "Escolaridade da Mãe", bolsas do tipo "Auxílio" e "Intercâmbio" apresentaram efeito significativo, mas com coeficientes padronizados muito baixos. 


\section{Discussão}

O modelo de explicação do rendimento acadêmico de estudantes de licenciatura testado nesse estudo avança em relação aos demais, que em sua maioria analisam a relação de uma variável, pois tenta ajustar um modelo estatístico que considere uma realidade mais complexa, em que inclui variáveis de contexto socioeconômico, assim como variáveis do contexto institucional dos estudantes.

A influência de variáveis socioeconômicas no desempenho acadêmico no ensino superior tem amplamente investigadas no Brasil e em outros países. Em um estudo de revisão de literatura foram identificadas muitas variáveis, tais como características individuais dos estudantes assim como características da instituição de ensino, que podem estar associadas positivamente ao desempenho acadêmico de estudantes do Ensino Superior (Almeida e Lopes, 2020). Esses estudos não têm se debruçado especificamente a estudantes em cursos de formação de professores. Alguns têm apontado que condições econômicas desfavoráveis tendem a afetar negativamente o desempenho em exames educacionais (García-González e Skrita, 2019; Rodrigues et al., 2017; Araújo, 2017; Thiele et al., 2016; Crepalde e Silveira, 2016; Oliveira e Melo-Silva, 2010; Carvajal, 2009), indicando que as estruturas sociais podem exercer forte influência sobre o comportamento individual (Nogueira e Nogueira, 2002; Wacquant, 2013).

Neste estudo, a variável "Cor-Raça Branca" também apresentou significativa explicação do rendimento acadêmico. Alguns estudos também apresentam diferenças de rendimentos quando comparado pela cor ou raça dos estudantes (Medeiros Filho, 2019; Rocha et al., 2018; Rodrigues et al., 2015; Silva et al., 2020). Nesses estudos, melhores desempenhos foram identificados em pessoas de cor ou raça branca.

Isso pode ser em decorrência de desigualdade sociais ainda presentes no Brasil. Dados do Instituto Brasileiro de Geografia e Estatística (IBGE, 2019) apontam diferenças relevantes entre populações brancas e negras. Esses dados indicam que maioria dos cargos gerenciais (68.6\%) são de pessoas brancas. Revela também que $41.7 \%$ das pessoas que vivem com renda abaixo da linha da pobreza são pretos ou pardos, enquanto que $19 \%$ são brancos. No que se refere a questões educacionais, a taxa de analfabetismo entre os pretos ou pardos é de $9.1 \%$, enquanto que entre os brancos é de $3.9 \%$.

Nesse sentido, a cor ou raça branca associada ao maior rendimento acadêmico no ensino superior pode ser por conta da condição histórica, cultural e social que tivemos no país, em que a educação formal foi melhor acessada por grupos elitizados economicamente, constituídos de 
pessoas de cor ou raça predominantemente branca. Enquanto que, para pessoas desfavorecida economicamente o acesso à educação se deu tardiamente.

Embora isso tenha se constituído como um problema histórico no contexto brasileiro, algumas políticas foram implementadas com o objetivo de mudar esse quadro. Dentre essas, reserva de vagas para cursos nas instituições de ensino superior para pessoas de baixa renda, negros e pessoas com deficiência no Sistema de Seleção Unificada (Sisu). Também, o Programa Universidade para Todos (Prouni) tem como finalidade conceder bolsas integrais e parciais em instituições privadas para os candidatos do Exame Nacional do Ensino Médio (Enem) (Brasil, 2005).

Outro dado importante apontado por esta pesquisa é a influência positiva do recebimento de bolsa de "Pesquisa" no rendimento acadêmico. Os resultados de algumas pesquisas (Costa, 2019; Araujo et al., 2018; Araújo, 2017) no Brasil são harmônicos a essa evidência, apresentando melhor rendimento os estudantes que receberam algum tipo de bolsa durante a graduação.

Quando analisado especificamente os estudantes de licenciatura a evidência permanece. Apresentam melhores resultados os estudantes bolsistas de políticas como Pibid comparados com os não bolsistas (Araujo et al., 2018). O melhor rendimento de bolsistas pode estar relacionado com as experiências que o exercício de bolsista exige, como por exemplo, contatos com a pesquisa, elaboração de projetos, a formação científica, traçando assim, caminhos diferenciados para esse grupo. Além do melhor rendimento, essas políticas, como o Programa Residência Pedagógicos (PRP), que proporciona bolsa para os estudantes realizarem o estágio na escola com o acompanhamento de professores experientes, proporciona uma reflexão positiva sobre a Educação por parte dos bolsistas (Costa et al., 2019).

Neste estudo a escolaridade do pai e da mãe explicou significativamente o rendimento acadêmicos dos estudantes, corroborando com os resultados de outros estudos (Rocha et al., 2019; García-González e Skrita, 2019; Costa, 2019; Brito, 2015) que identificaram significativa influência positiva no rendimento acadêmico de estudantes do ensino superior. Possivelmente pais mais escolarizados acompanham melhor o progresso acadêmico de seus filhos e possuem melhores condições econômicas para fornecer condições favoráveis de estudo.

Neste estudo, as variáveis de contexto testadas não conseguiram explicar parte importante da variância do desempenho acadêmico dos estudantes, restando ainda a maioria de sua variabilidade que é explicada por fatores desconhecidos. Possivelmente, variáveis psicológicas como a satisfação acadêmica (Osti et al., 2020), a adaptação ao curso, a motivação, estilos de aprendizagem, que não fizeram parte desse estudo, podem ser importantes na explicação do rendimento acadêmico (Araújo, 2017). Estudos longitudinais para o 
acompanhamento dessas variáveis também podem fornecer pistas importantes para a compreensão do desempenho acadêmico desses estudantes ao longo do curso. As características de entrada dos estudantes com seu perfil socioeconômico e objetivos de ingresso no curso (Rabelo et al., 2015) podem ser importantes preditores do rendimento dos estudantes ao longo do curso. Com isso, também seria possível compreender qual o perfil dos estudantes de alto desempenho e o que os distinguem dos de baixo desempenho acadêmico, o que permitiria direcionar esforços e estratégias para promover a aprendizagem desses estudantes.

\section{Conclusões}

Esta pesquisa partiu da problemática relacionada à compreensão dos contextos de aprendizagem dos estudantes dos cursos de formação de professores, e teve o objetivo de investigar se os fatores de contexto (socioeconômicos e institucionais) estão associados ao desempenho acadêmico de estudantes de licenciatura em matemática. Dessa forma, esta pesquisa indicou que as variáveis "Renda familiar" e o recebimento de bolsa "Pesquisa" pela instituição estão fortemente associados ao desempenho desses estudantes, quando mensurado pelo ENADE, corroborando com a hipótese explicitada no objetivo desta pesquisa. Outras variáveis como escolaridade dos pais, sexo dos estudantes e tipo de instituição apresentaram efeito significativo do desempenho, mas com coeficientes pequenos.

Os resultados dessa pesquisa indicam que não apenas as variáveis relacionadas a origem social dos estudantes, mas também variáveis institucionais são explicativas do seu rendimento acadêmico. Dito de outro modo, as instituições podem exercer papel importante no oferecimento de condições que induzem ao melhor aprendizado dos alunos. Nesse sentido, este estuda aponta que políticas educacionais de permanência e êxito, como por exemplo, bolsas acadêmicas, são relevantes para os estudantes.

\section{Referencias}

ÁlVARO PAGE, Mariano; BUENO MONREAL, María José; CALLEJA SOPEÑA, José Ángel; CERDÁN VICTORIA, Jesús; ECHEVERRÍA CUBILLAS, María José; GARCÍA LÓPEZ, Carmen; ...; \& TRILLO MARCO, Carmen (1990). Hacia un modelo causal del rendimiento académico. Centro de Publicaciones del Ministerio de Educación y Ciencia. Disponível em: https://redined. mecd.gob.es/xmlui/handle/11162/84655

ALMEIDA, Grasiany Sousa de; \& LOPES, Jayane Mara Rosendo (2020). O que explica o desempenho dos estudantes? Um estudo de revisão. Revista de Instrumentos, Modelos e Políticas em Avaliação Educacional, 1(1), e020004. https://doi.org/10.51281/impa.e020004 
ANDRIOLA, Wagner Bandeira (2009). Fatores institucionais associados aos resultados do exame nacional de desempenho estudantil (ENADE): estudo dos cursos de graduação da Universidade Federal do Ceará (UFC). REICE. Revista Iberoamericana sobre Calidad, Eficacia y Cambio en Educación, 7(1), 22-49. https://revistas.uam.es/index.php/reice/ article/view/5421

ARAUJO, Adriana Castro; ANDRIOLA, Wagner Bandeira; \& COELHO, Afrânio de Araújo (2018). Programa institucional de bolsa de iniciação à docência (Pibid): Desempenho de bolsistas versus não bolsistas. Educação em Revista, 34, 1-22. https://doi.org/10.1590/01024698172839

ARAUJO, Alexandra M (2017). Sucesso no Ensino Superior: Uma revisão e conceptualização. Revista de Estudios e Investigación en Psicología y Educación, 4(2), 132-141. https://doi. org/10.17979/reipe.2017.4.2.3207

ARAUJO, Fatima Helena Ramos de (2017). Determinantes do desempenho dos alunos das instituições de ensino superior brasileiras no ENADE 2013 [Dissertação de Mestrado, Universidade Federal do Ceará] Repositório Institucional UFC. http://www.repositorio. ufc.br/handle/riufc/29295

BARAHONA U., Planck (2014). Factores determinantes del rendimiento académico de los estudiantes de la Universidad de Atacama. Estudios Pedagógicos, 4O(1), 25-39. https://doi. org/10.4067/s0718-07052014000100002

BARBOSA, Glauber de Castro; FREIRE, Fátima de Souza; \& CRISÓSTOMO, Vicente Lima (2011). Análise dos indicadores de gestão das IFES e o desempenho discente no ENADE. Avaliação: Revista da Avaliação da Educação Superior (Campinas), 16(2), 317-344. https://doi.org/10.1590/S1414-40772011000200005

BARRIOS GAXIOLA, Melanie Itsel; \& FRÍAS ARMENTA, Martha (2016). Factores que influyen en el desarrollo y rendimiento escolar de los jóvenes de bachillerato. Revista Colombiana de Psicología, 25(1), 63-82. http://doi.org/10.15446/rcp.v25n1.46921

BATES, Douglas; MÄCHLER, Martin; BOLKER, Ben; \& WALKER, Steve (2015). Fitting linear mixedeffects models using Ime4. Journal of Statistical Software, 67(1), 1-48. https://doi.org/ 10.18637/jss.v067.i01

BAUER, Adriana; Cassettari, Nathalia; \& Oliveira, Romualdo Portela de (2017). Políticas docentes e qualidade da educação: uma revisão da literatura e indicações de política. Ensaio: Avaliação e Políticas Públicas em Educação, 25(97), 943-970. https://doi.org/10.1590/ s0104-40362017002501010

BRASIL (2018). Lei no 11.096, de 13 de janeiro de 2005. Brasília. http://www.planalto.gov.br/ ccivil_03/_ato2004-2006/2005/lei/l11096.htm 
BRITO, Tainá Fernandes de (2015). Corpo docente: fatores determinantes do desempenho discente no ENADE [Dissertação de Mestrado, Universidade de São Paulo] Biblioteca Digital de Teses e Dissertações da USP. https://doi.org/10.11606/D.12.2016.tde-21032016-115045

CAMARGO, Arlete Maria Monte de; \& MEDEIROS, Luciene das Graças de Miranda (2018). Expansão da educação superior, cursos de licenciatura e criação das novas universidades federais. Revista Educação em Questão, 56(47). https://doi.org/10.21680/19811802.2018v56n47ID14006

CARVAJAL, Aldo Colorado (2009). El capital cultural y otros tipos de capital en la definición de las trayectorias escolares universitarias. Anais do X Congreso Nacional de Investigación Educativa: sujetos de la educación. http://www.comie.org.mx/congreso/memoriaelectro nica/v10/contenido/contenido0116T.htm

CONSTA, Suzana Pilonetto (2019). Relação perfil socioeconômico e acadêmico e desempenho no ENADE (2017) da área de pedagogia (licenciatura) [Dissertação de Mestrado, Universidade Regional de Blumenau] Biblioteca Digital Brasileira de Teses e Dissertações. http://bdtd.ibict.br/vufind/Record/FURB_ab526a98228ba17647740643ffb61b12

COSTA, Eloiza Roza Dias da; SILVA, João Felix da; \& BENTO, Maria das Graças (2019). O Programa de residência pedagógica: Uma alternativa para a aproximação entre o acadêmico e o projeto político pedagógico da escola. Revista Multidisciplinar e de Psicologia, 13(48), 595-608. https://doi.org/10.14295/idonline.v13i48.2248

CREPALDE, Neylson João Batista Filho; \& SILVEIRA, Leonardo Souza (2016). Desempenho universitário no Brasil: Estudo sobre desigualdade educacional com dados do ENADE 2014. Revista Brasileira de Sociologia, 4(7). https://doi.org/10.20336/rbs.155

FIELD, Andy (2009). Descobrindo estatística usando o SPSS. ArtMed.

GARBANZO VARGAS, Guiselle María (2007). Factores asociados al rendimiento académico en estudiantes universitarios, una reflexión desde la calidad de la educación superior pública. Revista Educación, 31(1), 43-63. https://doi.org/10.15517/revedu.v31i1.1252

GARCÍA-GONZÁLEZ, Juan David; \& SKRITA, Anastasija (2019). Predicting academic performance based on students' family environment: Evidence for Colombia using classification trees. Psychology, Society,; \& Education, 11(3). http://doi.org/10.25115/psye.v11i3.2056

HAIR, Joseph F.; ANDERSON, Rolph E.; TATHAM, Ronald L; \& BLACK, Willian C (2005). Análise multivariada de dados. Artmed.

HOX, Joop; MOERBEEK, Mirjam; \& VAN DE SCHOOT, Rens (2017). Multilevel analysis: Techniques and applications. Routledge.

INSTITUTO BRASILEIRO DE GEOGRAFIA E ESTATÍSTICA - IBGE (2019). Desigualdades sociais por cor ou raça no Brasil: notas técnicas. IBGE. https://biblioteca.ibge.gov.br/index.php/ biblioteca-catalogo ?view $=$ detalhes $\&$ id $=2101681$ 
KER, H. W (2014). Application of hierarchical linear models / linear mixed-effects models in school effectiveness research. Universal Journal of Educational Research, 2(2), 173-180. https://doi.org/10.13189/ujer.2014.020209

KNOP, Márcia; \& Collares, Ana Cristina (2019). A influência da origem social na probabilidade de concluir os diferentes cursos de ensino superior. Sociedade e Estado, 34(2), 351-380. https://doi.org/10.1590/s0102-6992-201934020001

LAVOR, João Ferreira de; ANDRIOLA, Wagner Bandeira; \& LIMA, Alberto Sampaio (2015). Avaliando o impacto da qualidade da gestão acadêmica no desempenho dos cursos de graduação: um estudo em universidade pública brasileira. Revista Iberoamericana de Evaluación Educativa, 8(2), 233-254. https://revistas.uam.es/index.php/riee/article/view/2887

MARÔCO, João (2018). Análise estatística com o SPSS Statistics (5a ed). ReportNumber, Análise e Gestão da Informação.

MASCARENHAS, Suely; \& ROAZZI, Antonio (2015). Relações família-universidade, rendimento acadêmico e gênero no ensino superior brasileiro. Revista de Estudios e Investigación en Psicología y Educación, (05), 079-082. https://doi.org/10.17979/reipe.2015.0.05.272

MAUÉS, Olgaíses Cabral; \& CAMARGO, Arlete Maria Monte de (2014). A expansão do ensino superior, políticas de formação docente e atratividade da carreira. Revista Eletrônica de Educação, 8(1), 77-91. http://doi.org/10.14244/198271991013

MAUÉS, Olgaíses Cabral; \& SOUZA, Michele Borges de (2013). A expansão da educação superior no Brasil e as políticas de formação de professores. Cadernos de Educação, 45, 68-81. https://periodicos.ufpel.edu.br/ojs2/index.php/caduc/article/view/3823

MAUÉS, Olgaíses Cabral; SEGENREICH, Stella Cecilia Duarte; \& OTRANTO, Celia Regina (2015). As políticas de formação de professores: A expansão comprometida. Revista Educação em Questão, 51(37), 42-72. https://doi.org/10.21680/1981-1802.2015v51n37ID7171

MEDEIROS FILHO, Antonio Evanildo Cardoso de (2019). Percepção discente e desempenho dos estudantes dos cursos de licenciatura em educação física no ENADE 2017 [Dissertação de Mestrado, Universidade Estadual do Ceará] Repositório Institucional - UECE. https://sid uece. uece. br/siduece/trabalhoAcademicoPublico.jsf?id=84781

MEDEIROS FILHO, Antonio Evanildo Cardoso de; RODRIGUES, Yasmim S.; LOPES, Jayane Mara; \& Pontes Junior, José Airton de Freitas (2019). Fatores associados ao desempenho discente no exame nacional de desempenho dos estudantes (ENADE): Uma revisão integrativa. Revista Expressão Católica, 8(1), 87-96. https://doi.org/10.25190/rec.v8i1.2543

MOLCK, Adauto Marin; \& CALDERÓN, Adolfo Ignacio (2014). Exame nacional de desempenho de estudantes: Mapeamento e tendências temáticas da produção científica brasileira (20042010). Revista Educação Online, (15), 57-77. http://educacaoonline.edu.puc-rio.br/index. php/eduonline/article/view/55 
NOGUEIRA, Cláudio Marques Martins; \& NOGUEIRA, Maria Alice (2002). A sociologia da educação de Pierre Bourdieu: limites e contribuições. Educ. Soc., 23(78), 15-35. https://doi.org/10.1590/S0101-73302002000200003

OLIVEIRA, Anandra Santos Ribeiro de; \& SILVA, Ivair Ramos (2018). Indicadores educacionais no ensino superior brasileiro: Possíveis articulações entre desempenho e características do alunado. Avaliação: Revista da Avaliação da Educação Superior (Campinas), 23(1), 157177. https://doi.org/10.1590/s1414-40772018000100009

OLIVEIRA, Melina Del'Arco de; \& MELO-SILVA, Lucy Leal (2010). Estudantes universitários: A influência das variáveis socioeconômicas e culturais na carreira. Revista Semestral da Associação Brasileira de Psicologia Escolar e Educacional, 14(1), 23-34. https://doi.org/ 10.1590/s1413-85572010000100003

OSTI, Andreia; CHICO, Beatriz Marsili; OLIVEIRA, Vinícius de; \& ALMEIDA, Leandro S. (2020). Investigação de fatores relacionados à satisfação acadêmica no ensino superior brasileiro. Revista de Instrumentos, Modelos e Políticas em Avaliação Educacional, 1(3), e020015. https://doi.org/10.51281/impa.e020015

RABELO, Mauro Luiz; BISINOTO, Cynthia; MARINHO-ARAÚJO, Claisy; GRIBOSKI, Cláudia Maffini; \& MENEGHEL, Stela Maria (2015). Educação superior brasileira: perfil dos concluintes e sua percepção sobre a formação. Revista de Estudios e Investigación en Psicología y Educación, (14), 072-076. https://doi.org/10.17979/reipe.2015.0.14.869

REYES CARRETO, Ramón; GODÍNEZ JAIMES, Flaviano; ARIZA HERNÁNDEZ, Francisco Julián; SÁNCHEZ ROSAS, Francisco; \& TORREBLANCA IGNACIO, Omar Freddy (2014). Un modelo empírico para explicar el desempeño académico de estudiantes de bachillerato. Perfiles Educativos, 36(146), 45-62. http://www.scielo.org.mx/scielo.php?script=sci_arttext\&pid $=$ S0185-26982014000400004

ROCHA, Aline Lemes da Paixão; LELES, Claudio Rodrigues; \& QUEIROZ, Maria Goretti (2018). Fatores associados ao desempenho acadêmico de estudantes de nutrição no ENADE. Revista Brasileira de Estudos Pedagógicos, 99(251), 74-94. https://doi.org/10.24109/ 2176-6681.rbep.99i251.3162

RODRIGUES, Brenda Cristina de Oliveira; REZENDE, Marise Santana; MIRANDA, Gilberto José; \& PEREIRA, Janser Moura (2016). Determinantes do desempenho acadêmico dos alunos dos cursos de ciências contábeis no ensino a distância. Enfoque: Reflexão Contábil, 35(2), 139153. http://periodicos.uem.br/ojs/index.php/Enfoque/article/view/30105

RODRIGUES, Daniele Silva; SANTOS, Nálbia de Araújo; SANTANA, Monique da Silva Santana; \& LEMES, Ana Paula Moreira (2017). Diferenças entre gênero, etnia e perfil socioeconômico no exame nacional de desempenho do estudante do curso de ciências contábeis. UFSC, 14(33), 101-117. http://doi.org/10.5007/2175-8069.2017v14n33p101 
SILVA, Lucas Souza; SILVA, Francisca Feitosa da; \& MEDEIROS FILHO, Antonio Evanildo Cardoso de (2020). Raça/etnia e o desempenho dos estudantes de educação física no ENADE. Revista de Instrumentos, Modelos e Políticas em Avaliação Educacional, 1(3), e020016. https://doi.org/10.51281/impa.e020016

SILVA, Marjorie Cristina Rocha da; VENDRAMINI, Claudettte Maria Medeiros; \& LOPES, Fernanda Luzia (2010). Diferenças entre gênero e perfil sócio-econômico no exame nacional de desempenho do estudante. Avaliação: Revista da Avaliação da Educação Superior (Campinas), 15(3), 185-202. https://doi.org/10.1590/\$1414-40772010000300010

SOUZA, Alba Regina Battisti de; MARTINS FILHO, Lourival José; \& MARTINS, Rosa Elisabete Militz Wypyczynski (2020). Programa de Residência Pedagógica: conexões entre a formação docente e a Educação Básica. Formação Docente - Revista Brasileira de Pesquisa sobre Formação de Professores, 12(25), 137-150. https://doi.org/10.31639/rbpfp.v13i25.410

THIELE, Tamara; SINGLETON, Alexander; POPE, Danil; \& STANISTREET, Debbi (2016). Predicting students' academic performance based on school and socio-demographic characteristics. Studies in Higher Education, 41(8), 1424-1446. https://doi.org/10.1080/03075079. 2014.974528

WACQUANT, Loïc (2013). Bourdieu 1993: um estudo de caso em consagração científica. Revista Brasileira de Ciências Sociais, 28(83), 33-46. https://doi.org/10.1590/S0102-69092013000 300003

Fecha de recepción: 11 de febrero de 2021. Fecha de revisión: 26 de mayo de 2021. Fecha de aceptación: 29 de mayo de 2021. Fecha de publicación: 1 de julio de 2021. 